ISSN electrónico: 2445-1355

DOI: https://doi.org/10.14201/fj202162715

\title{
ADAPTACIÓN DE LA PFK1 EN CÉLULAS NERVIOSAS DE RATÓN EN CULTIVO PRIMARIO A LAS CONCENTRACIONES FISIOLÓGICAS DE OXÍGENO
}

\section{Adaptation of Pfk1 in Mouse Nerve Cells in Primary Crop to Physiological Concentrations of Oxygen}

\section{María MARTÍNEZ LUNA; Emilio FERNÁNDEZ SÁNCHEZ}

Instituto de Biología Funcional y Genómica. Grupo de investigación de Bioenergética y Estrés Oxidativo del Sistema Nervioso Central Laboratorio 2.7. Calle Zacarías González, 2. 37007 Salamanca.

Correo-e: mmrtnzluna@gmail.com*

RESUMEN: La glucolisis enlaza la necesidad de obtención de energía con el metabolismo, además, es la vía metabólica previa para que la mitocondria produzca la energía necesaria. La principal enzima reguladora de la glucolisis es la fosfofructoquinasa-1 (PFK1), cuya regulación es compleja. Esta enzima presenta tres isoformas: PFK1-L, PFK1-M y PFK1-C, cuyo nivel de expresión es específico de cada tejido. Este trabajo tiene como objetivo analizar el efecto que las concentraciones fisiológicas de $\mathrm{O}_{2}$, que son del $5 \%$, tienen sobre la actividad máxima y la cantidad total de la PFK1 como enzima reguladora de la glucolisis. Para ello se emplearon cultivos de neuronas y astrocitos en monocultivo y en cocultivo con concentraciones de $\mathrm{O}_{2}$ del $21 \%$ y el $5 \%$. Los resultados obtenidos muestran que los astrocitos presentan una actividad entre 4-5 veces mayor que las neuronas tanto en los monocultivos como en los cocultivos, además, su presencia provoca una mayor actividad en las neuronas tanto al $21 \%$ como al $5 \%$ de $\mathrm{O}_{2}$. El aumento de actividad en los monocultivos de las neuronas está relacionado con una mayor expresión de la PFK1-L, mientras que el de los cocultivos puede deberse al aumento de la expresión de la PFK1-L y la PFK1-M. 
Palabras clave: Fosfofructoquinasa-1 (PFK1); glucolisis; neuronas; astrocitos; $\mathrm{O}_{2}$.

ABSTRACT: Glycolysis links the need to obtain energy with metabolism, moreover, it's the previous metabolic pathway for the mitochondria to produce the necessary energy. The main glycolysis regulatory enzyme is phosphofructokinase-1 (PFK1) whose regulation is complex. This enzyme has three isoforms PFK1-L, PFK1-M and PFK1-C whose expression level is specific to each tissue. The objective of this work is to analyze the effect that physiological concentrations of $\mathrm{O}_{2}$, which are $5 \%$, have on the maximum activity and the total amount of PFK1 as a regulating enzyme of glycolysis. For this, neuron and astrocyte crop were used in monocrop and in cocrop with $\mathrm{O}_{2}$ concentrations of $21 \%$ and $5 \%$. The results obtained show that astrocytes presents an activity between 4-5 times greater than neurons both in monocrop and in cocrop, moreover, their presence causes greater activity in neurons at both $21 \%$ and $5 \% \mathrm{O}_{2}$. The increased activity in neuron monocrop is related to a higher expression of PFK1-L, while that of cocrop may be due to increased expression of PFK1-L and PFK1-M.

Keywords: Phosphofructokinase-1 (PFK1); glycolysis; neurons; astrocytes; $\mathrm{O}_{2}$.

\section{INTRODUCCIÓN}

El cerebro consume más del $20 \%$ del consumo total de oxígeno y glucosa a pesar de que solo representa un $2 \%$ del peso total corporal. Aunque en los pulmones la $\mathrm{pO}_{2}$ está en torno al 18-19\%, al cerebro llega una $\mathrm{pO}_{2}$ de alrededor del 12-14\%, pero las células, dependiendo de su distancia a los capilares sanguíneos, detectan una $\mathrm{pO}_{2}$ entre el 1 y el $10 \%$.

El metabolismo oxidativo de las células nerviosas es estrictamente dependiente de la disponibilidad de oxígeno, siendo la cadena respiratoria (CR) y la fosforilación oxidativa (FO) las principales vías de producción de energía metabólica en forma de ATP. Por su parte, la glucolisis es la principal vía metabólica de oxidación de glucosa, generando piruvato, ATP y NADH. En condiciones normales, cuando no hay suficiente oxígeno para producir el ATP necesario mediante la cadena respiratoria y la fosforilación oxidativa, se induce la glucolisis para producirlo, en lo que se conoce como glucolisis anaerobia con producción simultánea de lactato.

En la ruta glucolítica existen tres puntos de control regulados por distintas enzimas de las que nos centraremos en la fosfofructoquinasa-1 (PFK1) ya que es 
la principal enzima reguladora de la glucolisis y, además, presenta un mecanismo regulador complejo modulado por una gran variedad de metabolitos, fármacos y proteínas intracelulares (Sola-Penna et al., 2010).

La PFK1 presenta tres isoformas diferentes que reciben el nombre de PFK-M, PFK-L y PFK-C (P), cuyo peso molecular es de $85.2 \mathrm{kDa}, 85.0 \mathrm{kDa}$ y $85.6 \mathrm{kDa}$ respectivamente y sus genes se asocian a los cromosomas 1,21 y 10 respectivamente. Estas isoformas se asocian en forma de homo- o heterodímeros, homo- o heterotetrámeros o heteropolímeros y tienen un comportamiento alostérico. Se ha demostrado que la PFK-M y la PFK-L comparten el $68.6 \%$ de la secuencia de aminoácidos; la PFK-M y la PFK-C el $70.3 \%$, y la PFK-L y la PFK-C el $66.6 \%$. $\mathrm{La}$ afinidad por la fructosa-6-fosfato es mayor por la isoforma $\mathrm{M}$, luego la $\mathrm{L}$, por último, la C, mientras que la inhibición por ATP sigue el orden inverso. El nivel de expresión de las isoformas es específico de cada tejido, siendo el músculo esquelético el único que expresa una sola isoforma, la PFK-M, mientras que los demás tejidos expresan distintos niveles de las tres (Sola-Penna et al., 2010).

En el cerebro de una rata adulta la proporción de las tres isoformas es de PFK-C (3.9), PFK-L (1.4) y PFK-M (4.9). Sin embargo, la isoenzima $C$ es minoritaria en el feto y recién nacido, pero aumenta en el destete (Mhaskar, Dunaway, 1995).

La PFK1 es una enzima alostérica que se regula por metabolitos intermediarios (citrato) o productos finales del metabolismo celular como ATP, ADP, alanina, que regulan de manera alostérica la enzima (Sola-Penna et al., 2010).

La fructosa-2,6-bisfosfato (F2,6-BisP) es un metabolito que se sintetiza específicamente para regular la PFK1 y por este motivo es una regulación diferente a la de otros metabolitos, a pesar de que en ambos casos es alostérica. Se ha demostrado que este modulador favorece la formación de los tetrámeros y, por tanto, la forma activa de la PFK1 y revierte los efectos inhibidores del ATP. A su vez, la concentración de F2,6-BisP está regulada por la PFK2, que la sintetiza (Sola-Penna et al., 2010).

Herrero Méndez y colaboradores demostraron que las neuronas cultivadas al $21 \%$ de $\mathrm{O}_{2}$ presentan mRNA de la PFK2, pero no presentan concentraciones de dicha proteína, lo que nos indica que se está degradando, por lo que las concentraciones de F2,6-BisP son muy bajas y, por tanto, la actividad de la PFK1 en neuronas al $21 \%$ de $\mathrm{O}_{2}$ es muy baja (Herrero-Mendez et al., 2009).

\section{2. Овjetivo}

$\mathrm{La} \mathrm{pO}_{2}$ en las células nerviosas in vivo oscila entre el 1-10\% dependiendo de la distancia de la célula a los capilares sanguíneos. Lo que indica que por término medio la $\mathrm{pO}_{2}$ fisiológica del tejido nervioso es del $5 \%$. Sin embargo, tradicionalmente, los cultivos celulares se hacen con una $\mathrm{pO}_{2}$ atmosférica del $21 \%$, que no es fisiológica. 
Dado que la $\mathrm{pO}_{2}$ es esencial para el correcto funcionamiento de la CR y la FO y, por tanto, del metabolismo energético celular, el objetivo de nuestro trabajo consistió en analizar el efecto que las concentraciones fisiológicas de $\mathrm{O}_{2}$, que son del $5 \%$, tienen sobre la actividad máxima y cantidad total de la PFK1 como enzima reguladora de la glucolisis.

\section{Materiales y métodos}

Se utilizaron ratones p 57 recién nacidos o de un día de edad y embriones del decimoquinto día de la gestación, a los que se les extrajo el cerebro sin el bulbo olfatorio para realizar los cultivos primarios (el sacrificio de los animales fue realizado por un investigador en posesión de los permisos necesarios).

\subsection{Realización de los cultivos celulares}

Las células se incubaron durante seis días al $5 \%$ de $\mathrm{O}_{2}$ o al $21 \%$ de $\mathrm{O}_{2}$ y durante los últimos tres días se juntaron colocando los astrocitos en insertos dentro de las placas de cocultivos donde estaban las neuronas.

Los cultivos de neuronas y astrocitos se realizaron en días diferentes debido a que tienen protocolos diferentes.

\subsection{Preparación de las muestras}

Las células se lavaron dos veces con tampón fosfato salino (PBS) antes de la extracción de las muestras.

La extracción de proteínas celulares para su análisis por Western Blot (WB) se realizó lisando directamente las células en la placa de cultivo utilizando tampón RIPA frío $\left(4^{\circ} \mathrm{C}\right)$.

Los extractos celulares se trasvasaron a tubos eppendorf, se sometieron a ciclos de calentamiento a $100^{\circ} \mathrm{C}$ durante 5 minutos y de ultrasonidos (a $37 \mathrm{kHz}$ ) en un sonicador durante 10 minutos. Finalmente, se centrifugaron a $20.000 \mathrm{~g}$ durante 10 minutos y se guardaron a $-80^{\circ} \mathrm{C}$ hasta su utilización.

Para la extracción de las proteínas destinadas al análisis de la actividad de la PFK-1, las células lavadas se resuspendieron en $1 \mathrm{ml}$ de PBS trasvasándose la suspensión a tubos eppendorf. Se centrifugaron a $600 \mathrm{~g}$ durante 5 minutos eliminándose el sobrenadante y se lisaron con el tampón de extracción con tres ciclos de congelación-descongelación en nitrógeno líquido centrifugándose a continuación a $20.000 \mathrm{~g}$ y recogiendo el sobrenadante que se guardó a $-80^{\circ} \mathrm{C}$ para su posterior utilización. 


\subsection{Determinación de la actividad de la PFK1}

La determinación de la actividad de la PFK1 se realizó por espectrofotometría a $340 \mathrm{~nm}$ de longitud de onda midiendo la desaparición del NADH que se produce en reacciones acopladas con la PFK1 catalizadas por la aldolasa, triosa fosfato isomerasa y glicerol-3-fosfato deshidrogenasa.

\subsection{Determinación de proteinas (Roca et al., 2003)}

Las proteínas se determinaron por el método del ácido bicinconínico (BCA) a una longitud de onda de $562 \mathrm{~nm}$ según el protocolo descrito por el proveedor.

\subsection{Análisis de proteinas por Western Blot (Roca et al., 2003)}

Para el análisis de proteínas por WB se realizaron geles de poliacrilamida desnaturalizantes en presencia de SDS (SDS-PAGE).

1. Preparación de la muestra

Antes de la separación electroforética, las células se calentaron al baño María a $100^{\circ} \mathrm{C}$ durante cinco minutos en presencia de tampón de carga.

2. Electroforesis en gel

Las electroforesis se realizaron en geles de poliacrilamida al $8 \%$ de 1,5 $\mathrm{mm}$ de grosor a $100 \mathrm{~V}$ durante dos horas a temperatura ambiente. En los geles se cargaron entre 25 y $35 \mu \mathrm{g}$ de proteína de cada muestra.

3. Transferencia gel-membrana

Terminada la electroforesis las muestras se transfirieron a membranas de nitrocelulosa de $0,22 \mu \mathrm{m}$ a $400 \mathrm{~mA}$ de intensidad de corriente a $4{ }^{\circ} \mathrm{C}$ durante una hora y media.

4. Inmunodetección

Las membranas se bloquearon con leche desnatada al $5 \%$ en TTBS. Las incubaciones con los Ac primarios y secundarios se realizaron con leche desnatada al $2 \%$ en TTBS en proporción 1:1000 durante 24 h en frío y 2 $\mathrm{h}$ a temperatura ambiente respectivamente.

\section{Selección de anticuerpos}

Los Ac primarios contra la PFK1-L y la PFK1-M se prepararon en el laboratorio.

El Ac primario contra la PFK1-C se obtuvo comercialmente de la empresa Santa Cruz Biotechnology.

Los Ac secundarios utilizados contra la PFK1-L y la PFK1-M estaban ligados a la peroxidasa de rábano y la señal se reveló mediante la utilización 
de un sustrato quimioluminiscente cuya señal se capturó en una película fotográfica.

Por cuestiones de sensibilidad, para la detección de la PFK1-C se utilizó un Ac secundario fluorescente detectando la señal por fluorescencia infrarroja con el aparato FusionFX de VILBER a $800 \mathrm{~nm}$.

El análisis de las imágenes obtenidas en los WB se realizó utilizando los programas informáticos Image Lab (Bio-Rad) e ImageJ (National Institutes of Health).

\section{Resultados}

\subsection{Determinación de la actividad máxima de la PFK1}

\section{Actividad neuronas (nmoles)}

\section{Actividad astrocitos (nmoles)}

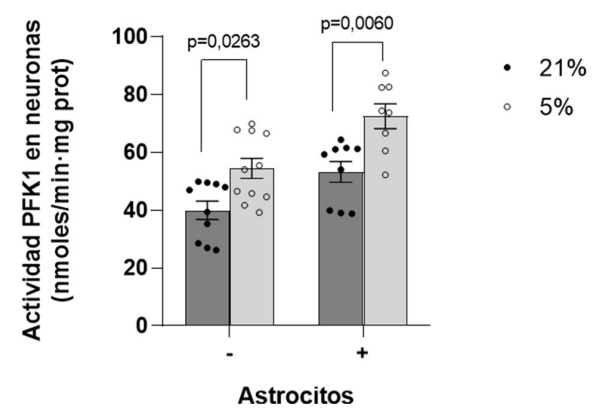

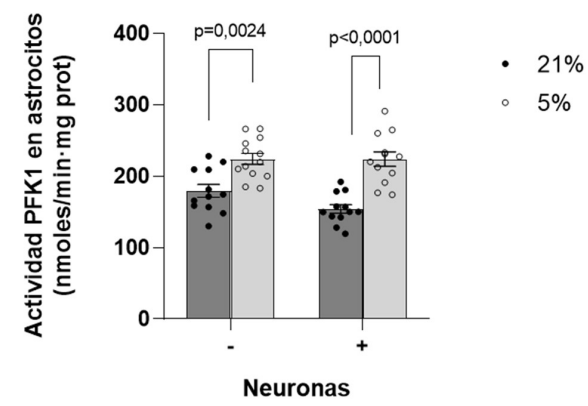

Figura 1. Actividad máxima de la PFK1 en cultivos primarios al $21 \%$ y al $5 \%$ de $\mathrm{O}_{2}$, en monocultivos y cocultivos. La actividad se determinó conforme se indica en el apartado 3.3 de materiales y métodos.

Todos los análisis estadísticos se han realizado con el ANOVA de dos vías utilizando el post test de Bonferroni.

Los resultados muestran que la realización de los cultivos al $5 \%$ de $\mathrm{O}_{2}$ produjeron un incremento significativo de la actividad enzimática entre un 20-30\% con respecto a los cultivos realizados al $21 \%$ de $\mathrm{O}_{2}$.

Además de lo ya indicado en el pie de figura, nuestros resultados sugieren que los astrocitos presentan una actividad de PFK1 entre 4-5 veces mayor que en las neuronas y, además, esta aumentó tras la incubación de las células al $5 \%$ de $\mathrm{O}_{2}$ tanto en los monocultivos como en los cocultivos. 
Igualmente, se observó que la presencia de astrocitos provoca una mayor actividad de la PFK1 en las neuronas tanto al $21 \%$ como al $5 \%$ de $\mathrm{O}_{2}$. Sin embargo, cuando los astrocitos se incuban en presencia de neuronas no se observan cambios en la actividad.

\subsection{Determinación de la cantidad de las distintas isoformas de la PFK1}

Después del revelado de las membranas obtenidas por WB se observaron las siguientes imágenes:

PFK1-L NEURONAS
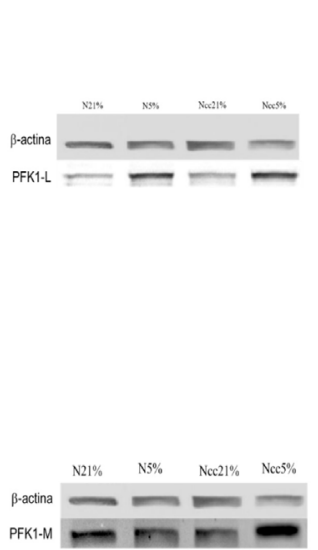

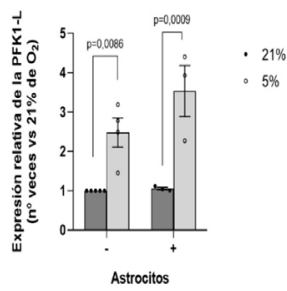

PFK1-M NEURONAS

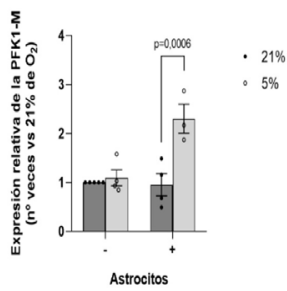

PFK1-L ASTROCITOS
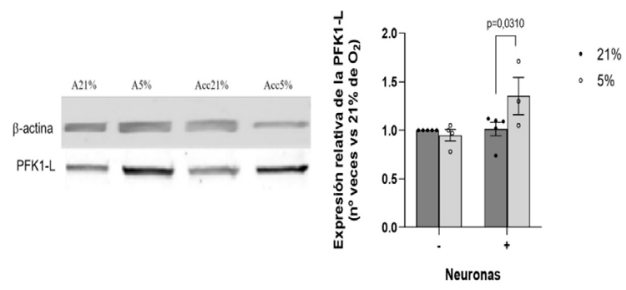

PFK1.M ASTROCITOS

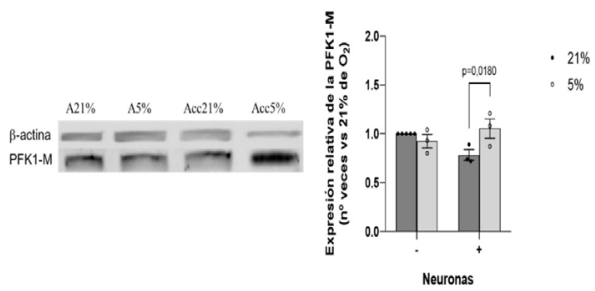

Figura 2. Expresión relativa de la PFK1-L en neuronas al $21 \%$ y $5 \% \mathrm{O}_{2}$ en presencia y ausencia de astrocitos (A). La incubación de las neuronas al $5 \%$ de $\mathrm{O}_{2}$ produjo un incrementos de PFK1-L en 2,5 y 3,5 veces en ausencia y presencia de astrocitos respectivamente, con respecto al $21 \%$ de $\mathrm{O}_{2}$.

Expresión relativa de la PFK1-L en astrocitos al $21 \%$ y $5 \% \mathrm{O}_{2}$ en presencia y ausencia de neuronas (B). La incubación de las neuronas al $5 \%$ de $\mathrm{O}_{2}$ produjo un incrementos de PFK1-L en 1,5 veces en ausencia y presencia de astrocitos respectivamente, con respecto al $21 \%$ de $\mathrm{O}_{2}$.

Expresión relativa de la PFK1-M en neuronas al $21 \%$ y $5 \% \mathrm{O}_{2}$ en presencia y ausencia de astrocitos (C). La incubación de las neuronas al $5 \%$ de $\mathrm{O}_{2}$ produjo un incrementos 
de PFK1-M de aproximadamente 2,3 veces en presencia de astrocitos, con respecto al $21 \%$ de $\mathrm{O}_{2}$.

Expresión relativa de la PFK1-M en astrocitos al $21 \%$ y $5 \% \mathrm{O}_{2}$ en presencia y ausencia de neuronas (D). La incubación de las neuronas al $5 \%$ de $\mathrm{O}_{2}$ produjo un incrementos de PFK1-M de aproximadamente 1,3 veces en presencia de neuronas, con respecto al $21 \%$ de $\mathrm{O}_{2}$.

En las neuronas, el aumento de actividad en los monocultivos está relacionado con una mayor expresión de la PFK1-L al no haberse detectado incrementos en las otras dos isoformas. Mientras que el incremento de la actividad en los cocultivos puede deberse al aumento de la expresión de la PFK1-L y PFK1-M.

PFK1-C ASTROCITOS
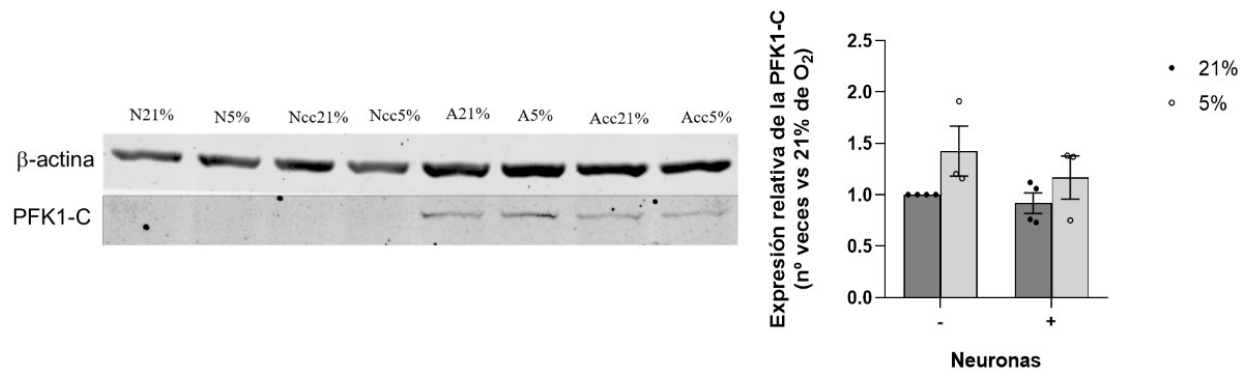

Figura 3. Expresión relativa de la PFK1-C en astrocitos al $21 \%$ y $5 \%$ de O2 en presencia y ausencia de neuronas.

No se detectaron diferencias con significatividad suficiente en la expresión de la PFK1-C entre los astrocitos cultivados al $21 \%$ y al $5 \%$ de $\mathrm{O}_{2}$, aunque se observa una tendencia a que la expresión al $5 \%$ de $\mathrm{O}_{2}$ es mayor que al $21 \%$ de $\mathrm{O}_{2}$.

Observando el incremento de actividad de los astrocitos al $5 \%$ de $\mathrm{O}_{2}$ frente a los cultivados al $21 \%$ de $\mathrm{O}_{2}$ visto en la figura y dado que no se observan diferencias en la PFK1-L y la PFK1-M probablemente la falta de significatividad en el incremento detectado en la cantidad de PFK1-C sea un mero artefacto. Sería necesario profundizar en este análisis para asegurar estos resultados.

En los astrocitos, el aumento de actividad en los cocultivos puede deberse a las isoformas PFK1-L y PFK1-M. 


\section{Conclusiones}

La actividad máxima de la PFK1 es mayor en condiciones fisiológicas que en condiciones del $21 \%$ de $\mathrm{O}_{2}$. Este resultado concuerda con los obtenidos en los análisis de WB de las diferentes isoformas de la PFK1. Además, la presencia de los astrocitos favorece que haya una mayor expresión de la enzima en las neuronas.

Por tanto, estos resultados sugieren que las células nerviosas a concentraciones fisiológicas de oxígeno presentan una mayor actividad glucolítica que en las condiciones estándar de cultivo sin perjuicio de la regulación metabólica a la que pueda estar sometida la PFK1.

\section{Bibliografía}

Dunaway GA, Kasten, TP. Nature of the rat brain 6-phosphofructo-1-kinase isozymes. J Biol Chem. 1985; 260(7):4180-4185.

Herrero-Mendez A. et al. The bioenergetic and antioxidant status of neurons is controlled by continuous degradation of a key glycolytic enzyme by APC/C-Cdh1. Nat Cell Biol. 2009; 11(6):747-752.

Mhaskar Y, Dunaway GA. Alteration of PFK subunit protein, synthesis, and mRNA during neonatal brain development. Elsevier. 1995; 85(1):54-57.

Nelson DL, Coz MM. Lehninger. Principios de bioquímica. 5. ${ }^{a}$ ed. Estados Unidos: Omega; 2017. pp. 527-538.

Roca P et al. Bioquímica. Técnicas y métodos. 1. a ed. España: Hélice; 2003. pp. 156-157; 168-170.

Rodwell VW et al. Harper's. Illustrated biochemistry. 30. ${ }^{a}$ ed. Estados Unidos: McGrawHill; 2009. pp. 168-175.

Sola-Penna $\mathrm{M}$ et al. Regulation of mammalian muscle type 6-phosphofructo-1-kinase and its implication for the control of the metabolism. IUBMB Life. 2010; 62(11):791-796.

Tanner LB et al. Four key steps control glycolytic flux in mammalian cells. Cell Syst. 2018; 7(1):49-62e8.

Webb BA et al. The glycolytic enzyme phosphofructokinase-1 assembles into filaments. J Cell Biol. 2017; 216(8):2305-2313. 
